University of Wollongong

Research Online

Australian Institute for Innovative Materials -

Papers

Australian Institute for Innovative Materials

2000

Microscopic treatment of substrate effects on linear and quadratic optical response of model Langmuir-Blodgett multilayers

Marc in het Panhuis

University of Wollongong, panhuis@uow.edu.au

R W. Munn

UMIST

Follow this and additional works at: https://ro.uow.edu.au/aiimpapers

Part of the Engineering Commons, and the Physical Sciences and Mathematics Commons

Research Online is the open access institutional repository for the University of Wollongong. For further information contact the UOW Library: research-pubs@uow.edu.au 


\title{
Microscopic treatment of substrate effects on linear and quadratic optical response of model Langmuir-Blodgett multilayers
}

\author{
Abstract \\ Microscopic calculations are reported of linear and quadratic nonlinear optical response in model \\ Langmuir-Blodgett films on a substrate, which is treated by the method of images. The effect of the \\ substrate is significant in the first two layers, and is greatest for tilted molecules with their head groups \\ adjacent to the substrate. The main qualitative effect is to lower the symmetry relative to a free-standing \\ film. Calculations for stearic acidfilms show that the substrate effect is most important for molecules \\ with nonuniform response on a metallic substrate.
}

\section{Keywords}

langmuir, blodgett, treatment, substrate, effects, linear, optical, response, microscopic, multilayers, quadratic, model

Disciplines

Engineering | Physical Sciences and Mathematics

\section{Publication Details}

in het Panhuis, M. \& Munn, R. W. (2000). Microscopic treatment of substrate effects on linear and quadratic optical response of model Langmuir-Blodgett multilayers. Journal of Chemical Physics, 113 (23), 10685-10690. 


\section{AD $\mid \begin{aligned} & \text { The Journal of } \\ & \text { Chemical Physics }\end{aligned}$}

\section{Microscopic treatment of substrate effects on linear and quadratic optical response of model Langmuir-Blodgett multilayers}

M. in het Panhuis and R. W. Munn

Citation: The Journal of Chemical Physics 113, 10685 (2000); doi: 10.1063/1.1323725

View online: $\mathrm{http}: / / \mathrm{dx}$.doi.org/10.1063/1.1323725

View Table of Contents: http://scitation.aip.org/content/aip/journal/jcp/113/23?ver=pdfcov

Published by the AIP Publishing

\section{Articles you may be interested in}

$(2+1)$-dimensional photonic crystals from Langmuir-Blodgett colloidal multilayers

Appl. Phys. Lett. 89, 043105 (2006); 10.1063/1.2234568

Analysis of linear and quadratic optical response of mixed Langmuir-Blodgett films of stearic acid and 5-CT

J. Chem. Phys. 113, 10691 (2000); 10.1063/1.1323724

Optical anisotropy of Langmuir-Blodgett sapphyrin films

Appl. Phys. Lett. 77, 3164 (2000); 10.1063/1.1324983

Microscopic calculations of linear and quadratic optical response in model Langmuir-Blodgett multilayers

J. Chem. Phys. 112, 6763 (2000); 10.1063/1.481238

Microscopic treatment of substrate effects on linear optical properties of model Langmuir-Blodgett films J. Chem. Phys. 101, 8159 (1994); 10.1063/1.468201

\section{AlP hameded}

Journal of Applied Physics is pleased to announce André Anders as its new Editor-in-Chief 


\title{
Microscopic treatment of substrate effects on linear and quadratic optical response of model Langmuir-Blodgett multilayers
}

\author{
M. in het Panhuis and R. W. Munn ${ }^{\text {a) }}$ \\ Department of Chemistry, UMIST, Manchester M60 1QD, United Kingdom
}

(Received 12 July 2000; accepted 19 September 2000)

\begin{abstract}
Microscopic calculations are reported of linear and quadratic nonlinear optical response in model Langmuir-Blodgett films on a substrate, which is treated by the method of images. The effect of the substrate is significant in the first two layers, and is greatest for tilted molecules with their head groups adjacent to the substrate. The main qualitative effect is to lower the symmetry relative to a free-standing film. Calculations for stearic acid films show that the substrate effect is most important for molecules with nonuniform response on a metallic substrate. (C) 2000 American Institute of Physics. [S0021-9606(00)70347-3]
\end{abstract}

\section{INTRODUCTION}

Langmuir-Blodgett films and other systems that comprise ordered molecular layers are of interest for developing materials with controllable structure and useful properties such as nonlinear optical response. Such materials are also often characterized optically, relying implicitly or explicitly on a suitable theory of the optical response. In a previous publication, ${ }^{1}$ we presented a comprehensive microscopic treatment of linear and quadratic optical response in model Langmuir-Blodgett multilayers. This treatment took account of molecular tilt and of nonuniform distribution of polarizability and hyperpolarizability within the molecules. However, for simplicity the treatment did not take account of the influence of the substrate on which Langmuir-Blodgett films are typically deposited. Substrate effects have been studied theoretically before, ${ }^{2-7}$ but only for linear response in a single monolayer. We have therefore sought to combine and complete the previous studies by providing a microscopic treatment of substrate effects on linear and quadratic optical response in model Langmuir-Blodgett multilayers.

For purposes of comparison our treatment here uses the same model structures as in our previous work, ${ }^{1}$ and as before $^{7}$ we use the method of images ${ }^{2-4}$ to incorporate the effect of the substrate, treating it as an isotropic dielectric. This approach suffices to explore how the substrate affects the different layers in thick films. It is known that the properties of Langmuir-Blodgett films vary as successive layers are deposited, with the first layer often differing significantly from subsequent ones. ${ }^{8}$ Such first-layer effects may well have structural origins, but they could also have a dielectric origin through the effect of the substrate, and calculations can explore this possibility. For quadratic nonlinear optical response, symmetry is a key factor, since such response is precluded in centrosymmetric structures. Discussions of this factor often concentrate on the symmetry of the film and ignore the substrate, but a film structure that is centrosym-

\footnotetext{
a) Author to whom correspondence should be addressed; electronic mail: R.W.Munn@umist.ac.uk
}

metric by itself loses that symmetry when the effect of the substrate is included.

Our primary purpose in presenting this work is to provide a complete systematic account of film response for model structures with model molecular responses. This is intended to bring out generic features likely to be useful in interpreting measurements or designing film structures. However, we also illustrate the usefulness of the treatment by applying it to a specific example, namely by showing how the choice of substrate material affects the local fields for monolayers of the classic Langmuir-Blodgett film forming species stearic acid (octadecanoic acid). For this purpose we use input polarizability data derived from the accompanying paper, ${ }^{9}$ which presents a detailed quantitative analysis of refractive index and optical second-harmonic generation (SHG) results reported ${ }^{6,10}$ for mixed monolayers formed between stearic acid and the molecule 5-CT (4"-n-pentyl4-cyano-p-terphenyl) that exhibits a thermotropic liquid crystal phase. The results were analyzed previously ${ }^{6}$ taking account of substrate effects by the method of images, but the molecules were treated as single points, whereas such elongated molecules imply the need for a submolecule treatment.

\section{THEORY}

The following treatment refers to a tetragonal structure, which contains one molecule in the unit cell with square packing. For a hexagonal structure with triangular packing, which has two molecules in the unit cell, the derivation is similar except for an additional subscript to label the different molecules in the unit cell. Previous work has shown little qualitative difference between these two packings. By construction, unit cell $\lambda$ in layer $g$ contains one molecule, composed of $s$ submolecules $j$, so that $(\lambda g j)$ defines a unique submolecule at position $\mathbf{r}_{\lambda g j}$. Translational symmetry within a layer means that molecular properties are independent of $\lambda$. The submolecules have polarizabilities $\boldsymbol{\alpha}_{g j}$ and first hyperpolarizabilities $\boldsymbol{\beta}_{g j}$ and the volume per molecule is $v$.

A uniform electric field $\mathbf{E}^{0}$ is applied to the film, consistent with applying a light wave of wavelength much greater 
than the unit cell edge and the film thickness. We first consider linear response. Submolecule $g j$ then acquires a dipole moment given by

$$
\mathbf{p}_{g j}=\boldsymbol{\alpha}_{g j} \cdot \mathbf{F}_{g j},
$$

where $\mathbf{F}_{g j}$ is the local polarizing electric field, which is independent of the unit cell $\lambda$. We assume that the multilayer structure consists of $N$ layers, in which case the linear polarization is given by

$$
\mathbf{P}^{(1)}=\sum_{g j} \mathbf{p}_{g j} / N v .
$$

This quantity relates the usual macroscopic electric field $\mathbf{E}$ to the applied field $\mathbf{E}^{0}$ through $^{11,12}$

$$
\mathbf{E}=\mathbf{E}^{0}-\mathbf{n}\left[\mathbf{n} \cdot \mathbf{P}^{(1)}\right] / \epsilon_{0},
$$

where $\mathbf{n}$ is the unit vector normal to the film. The polarization is also related to the macroscopic field through

$$
\mathbf{P}^{(1)}=\epsilon_{0} \boldsymbol{\chi}^{(1)} \cdot \mathbf{E},
$$

where $\boldsymbol{\chi}^{(1)}$ is the linear electric susceptibility, one of the targets of the present calculations. It yields the relative permittivity $\boldsymbol{\epsilon}_{r}$ as $\mathbf{1}+\boldsymbol{\chi}^{(1)}$, from which the refractive index $n_{e}$ in a direction defined by unit vector $\mathbf{e}$ is obtainable as

$$
1 / n_{e}^{2}=\mathbf{e} \cdot\left(\boldsymbol{\epsilon}_{r}\right)^{-1} \cdot \mathbf{e} \text {. }
$$

The effect of the substrate is taken into account by the method of images. Associated with each real dipole $\mathbf{p}$ at position $\mathbf{r}=(x, y, z)$ is an image dipole $\mathbf{p}^{I}$ at position $\mathbf{r}^{I}$ $=(x, y,-z)$, where the $z$-axis is defined as the outward normal to the substrate with the origin at the substrate. (Note that the results therefore depend on where the substrate is located relative to the layers.) The image dipole is given by

$$
\mathbf{p}^{I}=\mathbf{R} \cdot \mathbf{p},
$$

where the matrix $\mathbf{R}$ is given by

$$
\mathbf{R}=\left(\begin{array}{ccc}
-1 & 0 & 0 \\
0 & -1 & 0 \\
0 & 0 & 1
\end{array}\right) \rho,
$$

with $\rho$ the dielectric contrast given by $\left(\epsilon_{s}-1\right) /\left(\epsilon_{s}+1\right)$ for a substrate of isotropic relative permittivity $\epsilon_{s}$. The image dipoles contribute to the total fields that the real dipoles experience, since the local electric field is given by

$$
\mathbf{F}_{g j}=\mathbf{E}+\sum_{g^{\prime} j^{\prime}}\left[\mathbf{L}_{g j, g^{\prime} j^{\prime}} \cdot \mathbf{p}_{g^{\prime} j^{\prime}}+\mathbf{I}_{g j, g^{\prime} j^{\prime}} \cdot \mathbf{p}_{g^{\prime} j^{\prime}}^{I}\right] / \epsilon_{0} v N .
$$

Here $\mathbf{L}_{g j, g^{\prime} j^{\prime}}$ is a planewise Lorentz-factor tensor given by $^{11,12}$

$$
\mathbf{L}_{g j, g^{\prime} j^{\prime}}=\mathbf{T}_{g j, g^{\prime} j^{\prime}}+\mathbf{n n} \delta_{g g^{\prime}},
$$

where $\mathbf{T}_{g j, g^{\prime} j^{\prime}}$ is the real dimensionless planewise dipole tensor sum

$$
\mathbf{T}_{g j, g^{\prime} j^{\prime}}=\left.\frac{v}{4 \pi} \sum_{\substack{\lambda^{\prime} \\\left(\lambda g \neq \lambda^{\prime} g^{\prime}\right)}} \nabla \nabla\left(\frac{1}{r}\right)\right|_{\mathbf{r}=\mathbf{r}_{\lambda g j^{\prime}}-\mathbf{r}_{\lambda^{\prime} g^{\prime} j^{\prime}}},
$$

and $\mathbf{I}_{g j, g^{\prime} j^{\prime}}$ is the image dimensionless planewise dipole tensor sum

$$
\mathbf{I}_{g j, g^{\prime} j^{\prime}}=\left.\frac{v}{4 \pi} \sum_{\lambda^{\prime}} \nabla \nabla\left(\frac{1}{r}\right)\right|_{\mathbf{r}=\mathbf{r}_{\lambda g j^{-}}-\mathbf{r}_{\lambda^{\prime} g^{\prime} j^{\prime}}^{I}} .
$$

These sums are independent of $\lambda$ because translational symmetry ensures that the summand depends only on the difference $\lambda-\lambda^{\prime}$. The restriction on the real sum $\mathbf{T}_{g j, g^{\prime} j^{\prime}}$ excludes contributions from coincident points $\lambda g=\lambda g^{\prime}$ with $j=j^{\prime}$ and from different submolecules on the same molecule $\lambda g$ $=\lambda g^{\prime}$ with $j \neq j^{\prime}$, since a molecule does not polarize itself. In the image sum $\mathbf{I}_{g j, g^{\prime} j^{\prime}}$, no restrictions are required, because the real and image dipoles never coincide or belong to the same molecule.

Substitution in Eq. (8) for the local fields from Eqs. (1) and (6) gives a set of equations that can be solved to relate the local fields to the macroscopic field. The result can be expressed as

$$
\mathbf{F}_{g j}=\mathbf{d}_{g j} \cdot \mathbf{E},
$$

where $\mathbf{d}_{g j}$ is the local-field tensor given by

$$
\mathbf{d}_{g j}=\sum_{g^{\prime} j^{\prime}} \mathbf{D}_{g j, g^{\prime} j^{\prime}}=\sum_{g^{\prime} j^{\prime}}(\mathbf{J}-\mathbf{M} \cdot \mathbf{A})_{g j, g^{\prime} j^{\prime}}^{-1}
$$

Here the matrices on the right-hand side are of order $3 \mathrm{Ns}$, where $N$ is the number of layers and $s$ is the number of submolecules per molecule. The quantity $\mathbf{J}$ is the unit matrix with $3 \times 3$ submatrices $\mathbf{1} \delta_{g g^{\prime}} \delta_{j j^{\prime}} ; \mathbf{M}$ is a combined Lorentzfactor matrix ${ }^{2}$ with $3 \times 3$ submatrices $\mathbf{L}_{g j, g^{\prime} j^{\prime}}+\mathbf{I}_{g j, g^{\prime} j^{\prime}} \cdot \mathbf{R}$; and $\mathbf{A}$ is the polarizability matrix with $3 \times 3$ submatrices $\mathbf{a}_{g j} \delta_{g g^{\prime}} \delta_{j j^{\prime}}$, where $\mathbf{a}_{g j}$ is the dimensionless reduced polarizability $\boldsymbol{\alpha}_{g j} / \epsilon_{0} v N$. From Eqs. (2), (4), and (12), the linear susceptibility can be expressed as

$$
\boldsymbol{\chi}^{(1)}=\sum_{g j} \mathbf{a}_{g j} \cdot \mathbf{d}_{g j},
$$

which with Eq. (13) leads to

$$
\boldsymbol{\chi}^{(1)}=\sum_{g j, g^{\prime} j^{\prime}}\left(\mathbf{A}^{-1}-\mathbf{M}\right)_{g j, g^{\prime} j^{\prime}}^{-1}
$$

These results are developments of those derived previously, ${ }^{1,2,11,12}$ with the macroscopic electric field now defined for the whole film rather than for each individual layer (or for a monolayer film). As a consequence, the results are algebraically the same as for bulk dielectric response in molecular crystals, with an extra factor $N^{-1}$ and $g$ replacing the usual index $k$ for different molecules in the unit cell. In practice, it proves convenient to utilize this similarity by treating the $N$ layers of simple unit cells as one layer of composite unit cells containing $N$ molecules, one for each actual layer. 
We now consider quadratic response. This leads to a quadratic polarization $\mathbf{P}^{(2)}$ that is related to the macroscopic electric field by

$$
\mathbf{P}^{(2)}=\epsilon_{0} \boldsymbol{X}^{(2)}: \mathbf{E E},
$$

where $\boldsymbol{\chi}^{(2)}$ is the quadratic electric susceptibility, the other target of the present calculations. The treatment of linear response just outlined is readily extended to the previous treatment of nonlinear response ${ }^{13}$ to yield

$$
\boldsymbol{\chi}^{(2)}=\sum_{g j} \mathbf{b}_{g j} \vdots \mathbf{d}_{g j} \mathbf{d}_{g j} \mathbf{d}_{g j},
$$

where $\mathbf{b}_{g j}=\boldsymbol{\beta}_{g j} / \epsilon_{0} v N$ is a reduced first hyperpolarizability.

\section{NUMERICAL CALCULATIONS}

\section{A. Model}

We use the same model structures as in our previous work. ${ }^{1}$ They comprise ordered layers of molecules treated as a string of five spherical beads or submolecules, one of which (the head) can have a higher polarizability and hyperpolarizability from the others (the tail). The molecules may tilt by an angle $\theta$ away from the normal to the substrate, which is defined to be the $z$-axis, with the plane of tilt defining the $x$-axis. In X-type multilayer films, the molecules in all layers have their tails pointing downward, towards the substrate, while in Z-type multilayers, the molecules have their tails pointing upward, away from the substrate. In Y-type multilayers, the molecules have their tails pointing alternately upward and downward, but two types $\mathrm{Y}$ and $\mathrm{Y}^{\prime}$ are possible depending on whether the molecules in the first layer have their tails pointing upward or downward. In films composed of layers of tilted molecules, we consider two different packings. In parallel packing the molecules in successive layers lie with their axes parallel (antiparallel in Y-type films), while in herringbone packing the molecules in successive layers lie with their axes tilted by equal and opposite angles. Thus each type of tilted multilayer film requires a subscript $p$ or $h$ to denote the packing of the layers, e.g., $\mathrm{X}_{p}$.

The substrate is located at a distance $d$ below the center of the lowest submolecule. When this distance equals the radius $r$ of a submolecule, the substrate is tangential to the lowest submolecule. We restrict our treatment to the maximum dielectric contrast $\rho=1$, corresponding to infinite $\epsilon_{s}$, characteristic of a metal. Results are then compared with those for $\rho=0$, corresponding to a free-standing film with no substrate. This suffices to demonstrate the nature and the greatest possible magnitude of the effects caused by the substrate.

\section{B. Results}

In presenting our results, we concentrate on places where there are significant differences from the previous results with no substrate. ${ }^{1} \mathrm{We}$ also concentrate on the local field, which plays an important role in both $\boldsymbol{\chi}^{(1)}$ and $\boldsymbol{\chi}^{(2)}$, and on quadratic response, in which symmetry effects are important.

First we consider how the material response depends on the distance between the real layers and the substrate. Figure

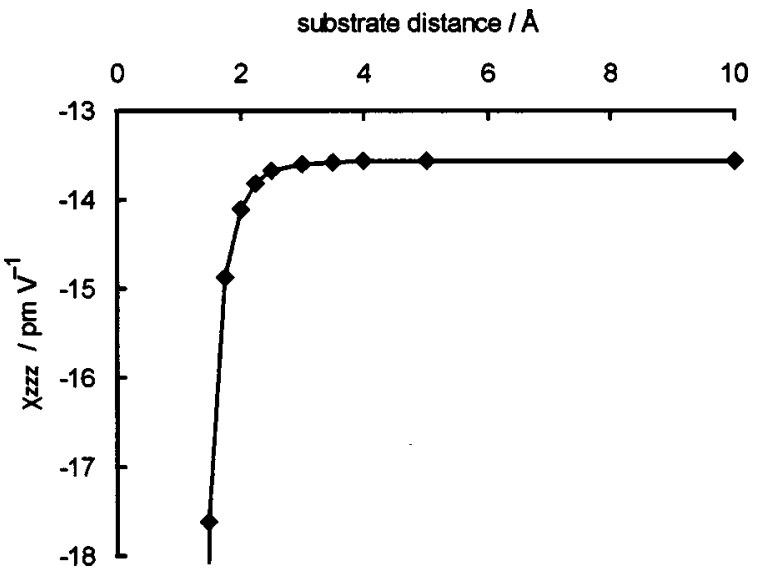

FIG. 1. Normal component $\chi_{z z z}$ of the quadratic susceptibility for an X-type Langmuir-Blodgett film consisting of two untilted layers on a metallic substrate for varying distances of the substrate plane from the center of the first submolecule. The value of $\chi_{z z z}$ without a substrate is $-13.57 \mathrm{pm} \mathrm{V}^{-1}$.

1 shows the normal component of the quadratic susceptibility for an X-type Langmuir-Blodgett film consisting of two untilted layers on a metallic substrate for varying distances of the substrate plane from the center of the first submolecule. For distances below $d=4 \AA$, the material response is unaffected by the presence of the substrate, but the response starts to vary noticeably for distances greater than this. In effect, interactions between real and image layers are significant only for one or two adjacent monolayers. All subsequent calculations use a distance $d=2.5 \AA$, corresponding to a substrate plane that lies tangential to the lowest submolecules and does produce significant effects.

Next we consider how the effect of the substrate on the material response depends on the number of layers. Figure 2 shows the normal component of the quadratic susceptibility for X-type untilted Langmuir-Blodgett films on a metallic substrate and with no substrate, as a function of the number of layers. As the number of layers increases, the two lines approach closely (note the large scale of the plot). Because the substrate effect extends significantly only to the first and

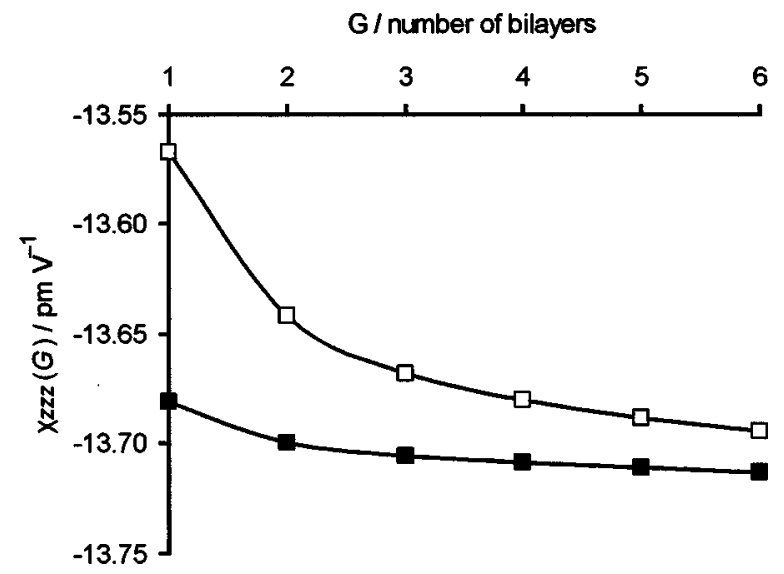

FIG. 2. The normal component $\chi_{z z z}$ of the quadratic susceptibility for X-type untilted Langmuir-Blodgett films on a metallic substrate (filled symbols) and with no substrate (open symbols), as a function of the number of bilayers. (The value for one bilayer with the substrate thus corresponds to the point for $d=2.5 \AA$ in Fig. 1.) 

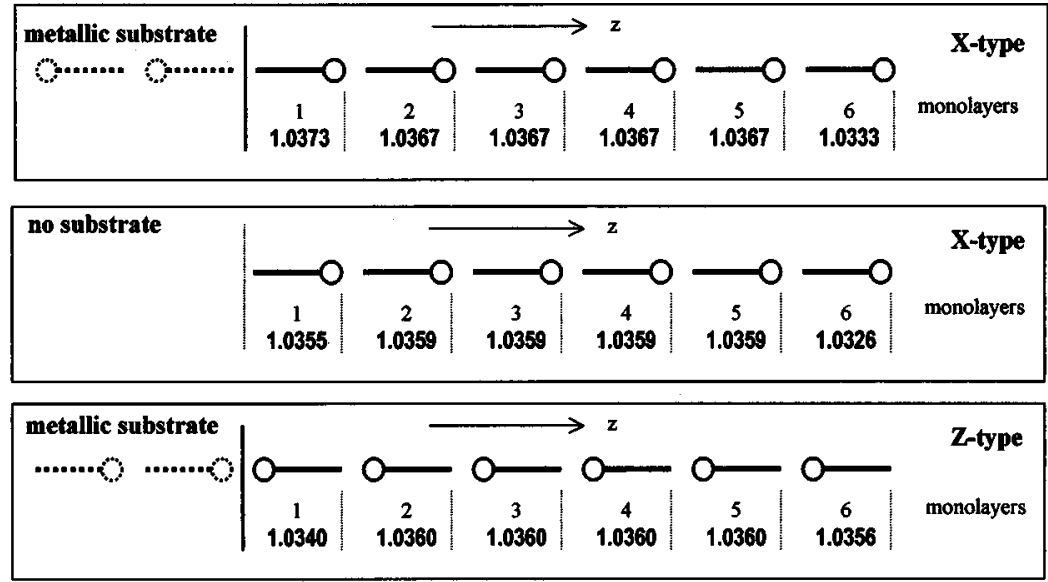

(a)
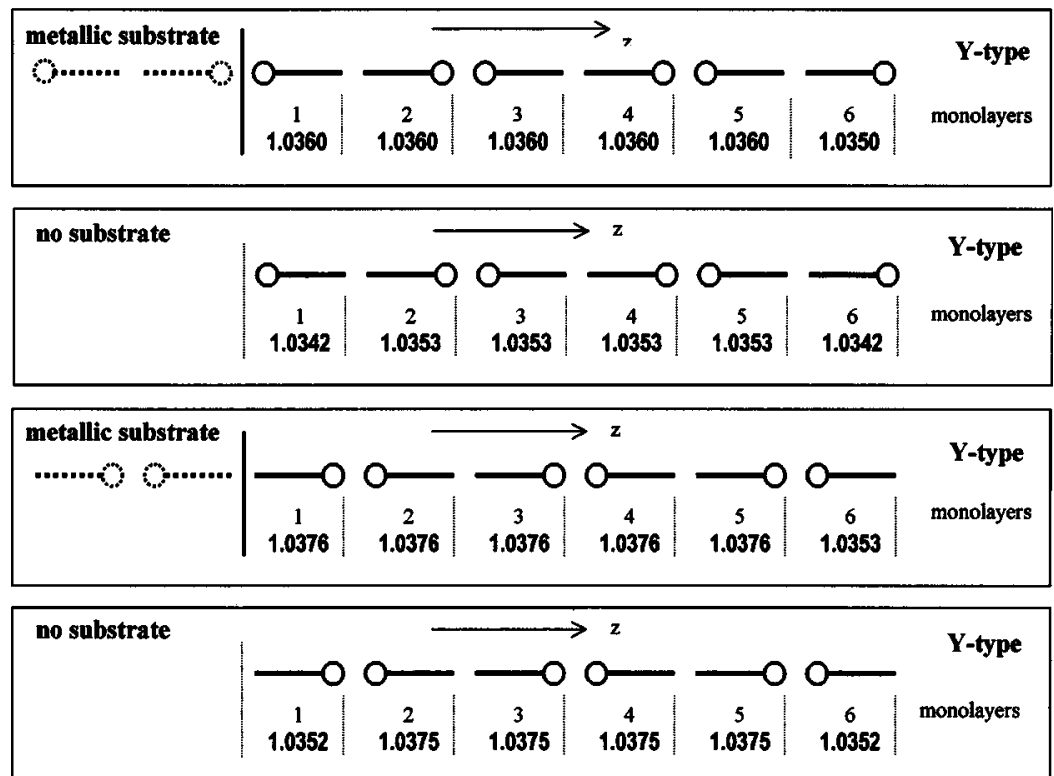

(b)

second monolayers, the net response of a thick material on a substrate will be equal to that of a free-standing thick material. In effect, the net response is a weighted average over all layers, and if only one or two layers out of many are affected by the substrate, then the response of the many unaffected by the substrate dominates.

We obtain more detailed insight into these effects by examining how the local field tensors vary in successive monolayers through a multilayer assembly. Figure 3 compares the normal component $d_{z z}$ of the layer local-field tensors in Langmuir-Blodgett films consisting of three untilted bilayers on a metallic substrate, in which case the image layers are equal in magnitude to the real ones. As usual only neighboring layers affect each layer significantly. In the absence of tilt the quantitative effects of the substrate on $d_{z z}$ are not large for these elongated molecules, but the qualitative effects are significant.

For X-type and Z-type films, the innermost layer (nearest the substrate) has a response different from that of the internal layers because it is the only layer affected significantly by the substrate. The effect is greater for the Z-type
FIG. 3. Normal component $d_{z z}$ of the layer local-field tensors in Langmuir-Blodgett films consisting of three untilted bilayers on a metallic substrate (on the lefthand side). In this case the image layers (indicated schematically to the left of the substrate plane) are equal in magnitude to the real ones. For each layer the figure shows the molecular orientation and the value of $d_{z z}$. film, and of opposite sign, since the highly polarizable group is adjacent to the substrate rather than remote from it as in the X-type film. On the other hand, the outermost layer (farthest away from the substrate) has a different response again, in this case because it has a neighboring layer only on one side. This result shows how interaction with the substrate leads to differentiation between X-type and Z-type films of molecules with distributed response, whereas free-standing $\mathrm{X}$-type and Z-type films have the same properties apart from the direction of the $z$-axis. Indeed, any distinction between these types arises only because of the substrate.

For Y-type and $\mathrm{Y}^{\prime}$-type films, on the other hand, the innermost bilayer has the same response as the middle bilayer, because the adjacent image layer for a metallic substrate gives them the same environment. However, the outermost layer still has a different response, because it has a neighboring layer only on one side. As a result, the Y-type and $\mathrm{Y}^{\prime}$-type films on a substrate lack a plane of symmetry, unlike the free-standing films. This means that even for untilted films the substrate induces a nonzero quadratic susceptibility $\boldsymbol{\chi}^{(2)}$. Nevertheless, there is a distinction between free- 
TABLE I. Local-field components $d_{x x}$ and $d_{z z}$ calculated for stearic acid monolayers on different substrates, assuming a uniform polarizability distribution.

\begin{tabular}{lcc}
\hline \hline Substrate & $d_{x x}$ & $d_{z z}$ \\
\hline None & 1.1934 & 1.0922 \\
Fused silica & 1.1955 & 1.1044 \\
Metal & 1.1994 & 1.1304 \\
\hline \hline
\end{tabular}

standing $\mathrm{Y}$-type and $\mathrm{Y}^{\prime}$-type films even in the absence of a substrate, because they have different numbers of head-tohead interactions.

This pattern of behavior carries over into films with tilted molecules, for which we do not present detailed results. The effects increase with increasing tilt but remain quite modest for the modest values of molecular response used here. For example, for two layers at $40^{\circ}$ tilt a metallic substrate changes the local-field components in the plane of tilt by about $3 \%$ for an $\mathrm{X}_{p}$ film and somewhat less for an $\mathrm{X}_{h}$ film. For six layers, the effect is reduced by a factor of two to three. Tilt also means that these types are distinct even in the absence of the substrate. Similar considerations apply for Y-type and $\mathrm{Y}^{\prime}$-type films.

\section{Stearic acid monolayers}

We now illustrate our treatment by applying it to monolayers of stearic acid (octadecanoic acid) on different substrates. For this purpose we use the following input data, derived in the accompanying article. ${ }^{9}$ The films are assumed to exhibit zero tilt, as indicated by $\mathrm{x}$-ray measurements on metal stearate films, ${ }^{8}$ which also indicate a thickness of about $25 \AA$. The estimated area per molecule of $19.8 \AA^{2}$, which for a square lattice implies a side of $4.44 \AA$. The molecule is treated as a set of ten submolecules equally spaced along its length, i.e., $2.5 \AA$ apart. The substrate is placed so that the real submolecule closest to it is also $2.5 \AA$ away from the image submolecule closest to it. Since stearic acid does not possess a very strong chromophore, the distribution of response within the molecules is approximated as uniform and the polarizability is approximated as axially symmetric. Then the polarizability volume along the molecular long $L$-axis is obtained from the monolayer refractive index on a fused silica substrate as $\alpha_{L L}=34.5 \AA^{3}$ and that across the axis is estimated as $\alpha_{M M}=14 \AA^{3}$. These values assume negligible substrate effect, which we shall justify below.

With these input data, we have calculated the two independent nonzero local-field components $d_{x x}$ and $d_{z z}$ for a free-standing film, for a film on a fused silica substrate (relative permittivity $\epsilon_{s}=2.122,{ }^{6}$ dielectric contrast $\rho=0.3594$ ), and for a film on a metallic substrate as in the preceding subsections. For uniform response, there is no distinction between X-type and Z-type films, for which we find the results given in Table I. In each case, $d_{x x}$ is much less affected by the substrate than $d_{z z}$. Compared with the free-standing film, the fused silica substrate makes only $0.2 \%$ difference in $d_{x x}$ and $1 \%$ in $d_{z z}$. This small effect is consistent with the previous assumption that it could be ignored in deducing the
TABLE II. Local-field components $d_{x x}$ and $d_{z z}$ calculated for X-type and Z-type stearic acid monolayers on different substrates, assuming an arbitrary nonuniform polarizability distribution.

\begin{tabular}{lcccccc}
\hline \hline \multirow{2}{*}{ Substrate } & \multicolumn{2}{c}{ X-type } & & \multicolumn{2}{c}{ Z-type } \\
\cline { 2 - 3 } \cline { 5 - 6 } \cline { 5 - 6 } & $d_{x x}$ & $d_{z z}$ & & $d_{x x}$ & $d_{z z}$ \\
\hline None & 1.1909 & 1.1111 & & 1.1909 & 1.1111 \\
Fused silica & 1.1921 & 1.1192 & & 1.2084 & 1.1474 \\
Metal & 1.1944 & 1.1358 & & 1.2496 & 1.2571 \\
\hline \hline
\end{tabular}

polarizability (especially since $\alpha_{M M}$ is estimated rather than directly calculated). Even the metallic substrate makes only $0.5 \%$ difference in $d_{x x}$ and $3.5 \%$ in $d_{z z}$.

Although we do not have information on the distribution of response within the stearic acid molecule, as already noted, we have explored the difference a highly nonuniform distribution would make to the local fields. For this purpose, we assume the same ratio of $6: 1$ between the head and tail polarizabilities as in the model calculations. There is then a distinction between X-type and Z-type films in the presence of a substrate. The results are given in Table II. They show smaller substrate effects for X-type films than for uniform polarizability, because the highly polarizable head is remote from the substrate and hence from the influence of the image dipoles. Conversely, the results show larger effects for Z-type films than for uniform polarizability, because the highly polarizable head is adjacent to the substrate and quite strongly influenced by the image dipoles. It is also noticeable that the anisotropy of the local field reduces from uniform response to nonuniform response and for nonuniform response from X-type to Z-type films.

\section{DISCUSSION AND CONCLUSIONS}

We have extended our previous treatment of linear and quadratic optical response of model Langmuir-Blodgett films to include the effect of a substrate, treated as a continuous dielectric in the image-dipole approximation. The major qualitative effects arise from the lowering of symmetry that the substrate produces. This makes layers inequivalent that are equivalent in free-standing multilayers. In particular, Y-type films are never strictly centrosymmetric once the substrate is considered, and hence should always have a nonzero quadratic susceptibility (which need not, however, be large). However, the substrate effects fall off rapidly for layers remote from the substrate, so that the aggregate response for thick multilayers is scarcely affected by the substrate. As illustrated by our calculations on stearic acid, monolayers can suffer significant effects on metallic substrates when the polarizability is markedly nonuniform and the most polarizable group is adjacent to the substrate, although a substrate reduces the anisotropy of the local-field tensor.

With its predecessor, ${ }^{1}$ this article completes our modeling of optical response in Langmuir-Blodgett films. We have treated linear and nonlinear response, for monolayers and multilayers of all types, tilted and untilted, with uniform and nonuniform response, with and without a substrate. We have used model molecular structures and responses and idealized periodic film structures, to provide a systematic over- 
view of how these factors contribute to the optical response of films. This should suffice for qualitative interpretation of measurements. However, detailed quantitative analysis is also possible, as illustrated in the accompanying article, ${ }^{9}$ which analyzes the linear and nonlinear response observed ${ }^{6}$ in mixed Langmuir-Blodgett monolayers of stearic acid and the mesogen 5-CT (4"-n-pentyl-4-cyano- $p$-terphenyl).

\section{ACKNOWLEDGMENTS}

We are grateful to J. F. McGilp for encouraging discussions. This work was supported under the EU TMR Network Program, contract FMRX-CT96-0047.
${ }^{1}$ M. in het Panhuis and R. W. Munn, J. Chem. Phys. 112, 6763 (2000).

${ }^{2}$ A. Bagchi, T. R. Barrera, and R. Fuchs, Phys. Rev. B 25, 7086 (1982).

${ }^{3}$ P. Ye and R. Shen, Phys. Rev. B 28, 4288 (1983).

${ }^{4}$ W. Chen and W. L. Schaich, Surf. Sci. 218, 580 (1989).

${ }^{5}$ L. M. Hayden, Phys. Rev. B 38, 3718 (1989).

${ }^{6}$ Z.-R. Tang, M. Cavanagh, and J. F. McGilp, J. Phys.: Condens. Matter 5, 3791 (1993); 5, 7903 (1993).

${ }^{7}$ R. W. Munn, J. Chem. Phys. 101, 8159 (1994).

${ }^{8}$ M. C. Petty, in Langmuir-Blodgett Films, edited by G. G. Roberts (Plenum, New York, 1990), p. 189.

${ }^{9}$ M. in het Panhuis and R. W. Munn, J. Chem. Phys. 113, 10691 (2000), following paper.

${ }^{10}$ Z.-R. Tang and J. F. McGilp, J. Phys.: Condens. Matter 4, 7965 (1992).

${ }^{11}$ R. W. Munn, J. Chem. Phys. 97, 4532 (1992).

${ }^{12}$ R. W. Munn, J. Mater. Chem. 4, 849 (1994).

${ }^{13}$ R. W. Munn and M. M. Shabat, J. Chem. Phys. 99, 10059 (1993). 